\title{
Spin-spin correlations of magnetic adatoms on graphene
}

\author{
A. D. Güçlü and Nejat Bulut \\ Department of Physics, Izmir Institute of Technology, IZTECH, TR-35430 Izmir, Turkey
}

(Received 2 November 2014; revised manuscript received 29 December 2014; published 3 March 2015)

\begin{abstract}
We study the interaction between two magnetic adatom impurities in graphene using the Anderson model. The two-impurity Anderson Hamiltonian is solved numerically by using the quantum Monte Carlo technique. We find that the interimpurity spin susceptibility is strongly enhanced at low temperatures, significantly diverging from the well-known Ruderman-Kittel-Kasuya-Yoshida result which decays as $R^{-3}$.
\end{abstract}

DOI: 10.1103/PhysRevB.91.125403

PACS number(s): 75.75.-c, 73.22.Pr

\section{INTRODUCTION}

Graphene [1-4], a two-dimensional honeycomb lattice of carbon atoms, shows promise as a material for nanoelectronics due to high electronic and thermal conductivity. Moreover, graphene structures engineered at the nanoscale are shown to give rise to unique magnetic properties due to the formation of finite magnetic moments at the edges [5-14], which could be important for nanoelectronic and spintronic device applications. Another way of probing magnetism in graphene is through the exchange interaction between impurity atoms mediated by the host electrons, known as the RudermanKittel-Kasuya-Yoshida (RKKY) interaction [15-20]. Understanding the effective interaction between impurity atoms in graphene is also important from a fundamental physics point of view since the excitations on a honeycomb lattice are massless Dirac fermions, giving rise to a behavior different from semiconductor or metal host structures [15-20].

The RKKY interaction in graphene exhibits unique features that are different from other two-dimensional systems. In Ref. [21], it was predicted that RKKY interactions should decay as $R^{-3}$, in contrast with $R^{-2}$ behavior found in a twodimensional electron gas [22], where $R$ is the distance between the two impurities. This was later confirmed in Ref. [17], where other important features of RKKY interactions in graphene were clarified as well. In particular, a general proof regarding the sign of the RKKY interaction in a half-filled bipartite lattice was given: The interaction between moments sitting on the same (opposite) sublattice(s) is ferromagnetic (antiferromagnetic). We note that the biparticity of the graphene lattice is also at the heart of Lieb's theorem on magnetism [23], which gives rise to edge magnetism in graphene nanostructures [12-14]. In Ref. [17], it was also shown that the RKKY interaction is subject to an oscillatory term of the type $1+\cos (2 \Delta \mathbf{k} \cdot \mathbf{R})$ ( $2 k_{F}$ oscillations), where $\Delta \mathbf{k}$ is the reciprocal lattice vector connecting two Dirac points in the Brillouin zone. All these features were confirmed by others [18-20] using different approximation schemes. It should be noted that impurities do not have to sit on top of a particular atom, but can bond with several neighboring atoms. In fact, the mechanical and electronic properties of various adatoms on graphene were previously investigated using ab initio methods [24-31]. For transition metal adatoms, most calculations predict that the plaquette configuration (where the impurity atom bonds with the six carbon atoms of a hexagon in the honeycomb lattice) is the most stable. However, generalized gradient approximation
(GGA) calculations [28] including Hubbard on-site interaction parameter $U$ show that, the on-top configuration may become more stable for $U>4 \mathrm{eV}$. On the other hand, according to RKKY analysis [17], the interaction between plaquette-type impurities (where the impurity atom bonds with the six carbon atoms of a hexagon in the honeycomb lattice) is much weaker and decays rapidly as $R^{-4}$. This is also consistent with our quantum Monte Carlo (QMC) results (not shown). Therefore, in the following we will focus on the interaction between impurities with an on-top configuration. This situation is particularly relevant to transition metal adatoms with large $U$.

In this work, we use the Hirsch-Fye QMC method [32] to calculate the magnetic susceptibilities of the two-impurity Anderson model. One should note that, as opposed to the RKKY model where the spins are taken to be classical, the Anderson model can capture important effects of spin fluctuations. Moreover, the interactions are mediated by the Coulomb on-site term $U$, which is absent in the RKKY model. It is therefore interesting to compare the results of the two models. We find that, while the biparticity theorem of Ref. [17] and the $2 k_{F}$ oscillation behavior also emerge from the Anderson model, the long-range behavior of the effective RKKY interaction is strongly enhanced, becoming several orders of magnitude larger at longer distances.

\section{MODEL AND METHOD}

The two-impurity Anderson model for a graphene host is given by

$$
\begin{aligned}
H= & \sum_{\mathbf{k} \alpha \sigma} \epsilon_{\mathbf{k}} c_{\mathbf{k} \alpha \sigma}^{\dagger} c_{\mathbf{k} \alpha \sigma}+E_{d} \sum_{i \sigma} d_{i \sigma}^{\dagger} d_{i \sigma} \\
& +\sum_{\mathbf{k} \alpha i \sigma}\left(V_{\mathbf{k} \alpha i} c_{\mathbf{k} \alpha \sigma}^{\dagger} d_{i \sigma}+\text { H.c. }\right)+U \sum_{i} n_{i d \uparrow} n_{i d \downarrow},
\end{aligned}
$$

where $c_{\mathbf{k} \alpha \sigma}^{\dagger}$ creates a host electron with wave vector $\mathbf{k}$ and spin $\sigma$ in the valence $\alpha=v$ or conduction $\alpha=c$ band, $d_{i \sigma}^{\dagger}$ creates an electron at the impurity site $i$, and $n_{i d \sigma}=d_{i \sigma}^{\dagger} d_{i \sigma}$. In addition, $U$ is the on-site Coulomb repulsion and $E_{d}$ is the impurity energy level. The electronic spectrum of the graphene host $\epsilon_{\mathbf{k}}$ and the hybridization matrix elements $V_{\mathbf{k} \alpha i}$ are calculated analytically in terms of graphene structure factor $f(\mathbf{k})$ in the nearest neighbor approximation with hopping parameter $t$. The impurity-carbon atom hybridization parameter is denoted by $V$. The calculations are performed 
within the symmetric Anderson model where $E_{d}=-U / 2$, as a function of inverse temperature $\beta=1 / T$ and the distance between the two impurities $R$. Strictly speaking, transition metal adatoms have five $d$ orbitals contributing to the spin. In our simplified model, the degeneracy of the orbitals is assumed to be lifted so that only one of the orbitals couples strongly to the itinerant host electrons.

The numerical results presented here were obtained using the Hirsch-Fye quantum Monte Carlo technique which allows us to compute the Matsubara single-particle Green's functions for impurity sites $i$ and $j$,

$$
G_{i j}^{\sigma}(\tau)=-\left\langle T_{\tau} d_{i \sigma}(\tau) d_{j \sigma}^{\dagger}(0)\right\rangle,
$$

where $T_{\tau}$ is the Matsubara time-ordering operator and $d_{i \sigma}(\tau)=$ $e^{H \tau} d_{i \sigma} e^{-H \tau}$. In addition, we calculate the zero-frequency interimpurity magnetic susceptibility using

$$
\chi_{12}(\omega=0)=\int_{0}^{\beta} d \tau\left\langle M_{1}^{z}(\tau) M_{2}^{z}(0)\right\rangle,
$$

where $M_{i}^{z}=n_{i d \uparrow}-n_{i d \downarrow}$. The local magnetic moments of impurity adatoms on graphene were studied in Ref. [33]. Here we concentrate on the impurity-impurity magnetic correlations.

\section{RESULTS}

In Fig. 1, we consider the case where the two impurities are located along the zigzag direction of the honeycomb lattice, sitting on different [zigzag $A B$, Fig. 1(a)] and the same [zigzag $A A$, Fig. 1(b)] sublattices. The static magnetic susceptibilities $\chi_{12}$ given in Eq. (3) are calculated as a function of the distance between the impurities $R$ (in units of the second nearest neighbor distance $b$ ) at different inverse temperatures $\beta$ expressed in units of $t^{-1}$. We take $V=t$ and $U=0.8 t$ (see Fig. 2 for larger values of $U$ ). Here, the results are also compared to the analytical RKKY results [19], denoted by the dashed lines. For the $A B$ configuration, the RKKY model yields to an antiferromagnetic coupling between the two impurities, as seen from the sign of $\chi_{i j}$, and Fermi oscillations with minima at every $(2+3 n)$ th $B$ atom along the zigzag $A B$ direction. For the $A A$ configuration, the coupling is ferromagnetic and the oscillations have maxima at every $(3+3 n)$ th $A$ atom. For both cases, as already mentioned, the oscillations decay as $R^{-3}$. All these behaviors agree well with the Anderson model (QMC) results, especially at higher temperatures. However, the results are very sensitive to the temperature. As the temperature is lowered, significant deviations from the RKKY results occur. The overall magnitude of the static susceptibility increases by several orders, especially at larger $R$ values, the decay of RKKY becomes much slower, and the Fermi oscillations become less prominent. Strikingly, at $\beta=128 t^{-1}$, which corresponds to $T=272 \mathrm{~K}$ for $t=3 \mathrm{eV}$, there is no decay in the range of $R$ studied here.

In Fig. 2, we investigate the effect of $U$ on the static susceptibility. The susceptibilities are calculated at $\beta=32 t^{-1}$ and $\beta=64 t^{-1}$ for the zigzag $A B$ case [similar to Fig. 1(a) but on a linear scale instead of logarithmic]. Calculations are repeated for $U=0.8 t, 1.4 t$, and $2 t$, corresponding to $2.4,4.2$, and $6 \mathrm{eV}$, respectively. Although the exact value for $U$ is not known for $3 d$ transition metal adatoms in
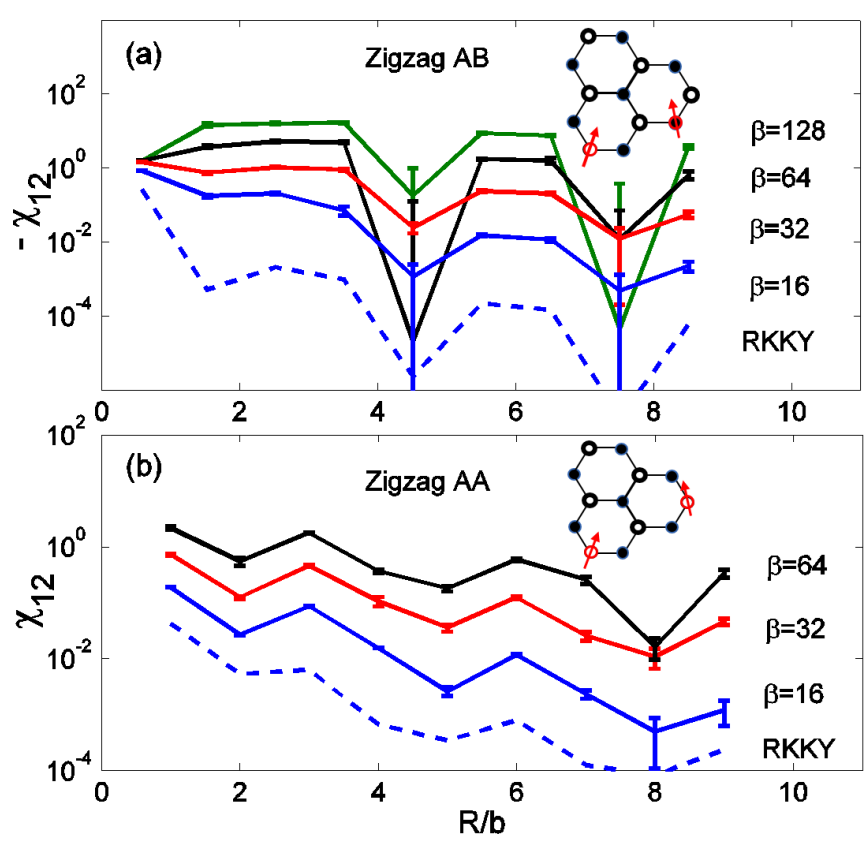

FIG. 1. (Color online) The static magnetic susceptibility between two magnetic adatom impurities along the zigzag direction as a function of distance for (a) the $A B$ configuration (impurities on opposite sublattices, shown in the inset) and (b) the $A A$ configuration (impurities on the same sublattice, shown in the inset), obtained by QMC calculations at different inverse temperatures $\beta$. The dashed lines are the RKKY results from Ref. [19]. The magnetic coupling obtained by the QMC shows the same ferromagnetic and antiferromagnetic behavior, and the $2 k_{F}$ oscillations as in the RKKY results. However, at low temperatures, the effective magnetic coupling becomes much stronger and the QMC results diverge from the RKKY's $R^{-3}$ decay.

graphene, its effective value is estimated to be in the range of 2-4 eV [27-30]. As the statistical fluctuations increase for larger values of $U$ in QMC calculations, the analysis is restricted to four different values of $R$ corresponding to first, third, sixth, and ninth atoms (along the zigzag direction) belonging to the maxima of the RKKY oscillations. Clearly, the main effect of increasing $U$ is to increase the susceptibility for $R / b<3$, i.e., at very short ranges. For $R / b>3$, we do not observe a significant change in $\chi_{i j}(R)$ within our statistical accuracy. The overall behavior thus becomes slightly closer in shape to the $R^{-3}$ decay (dashed curve), but there are still several orders of magnitude of difference between the RKKY and Anderson model results. Thus, the main conclusions of Fig. 1 remain unchanged for the values of $U$ considered here.

We now turn to the armchair configuration. In Fig. 3, the results are presented for $U=0.8 t$ at different values of $\beta$, for the $A B$ and $A A$ configurations. Again, the antiferromagnetic and ferromagnetic phases for the $A B$ and $A A$ configurations are consistent with the RKKY model. Note that along the armchair direction, the RKKY model does not exhibit Fermi oscillations. This is also consistent with the QMC results at higher temperatures (lower $\beta$ ) which show no clear structure within our statistical accuracy. As the temperature is lowered, similar to the zigzag case, the static susceptibility increases by more than two orders of magnitude at larger distances 


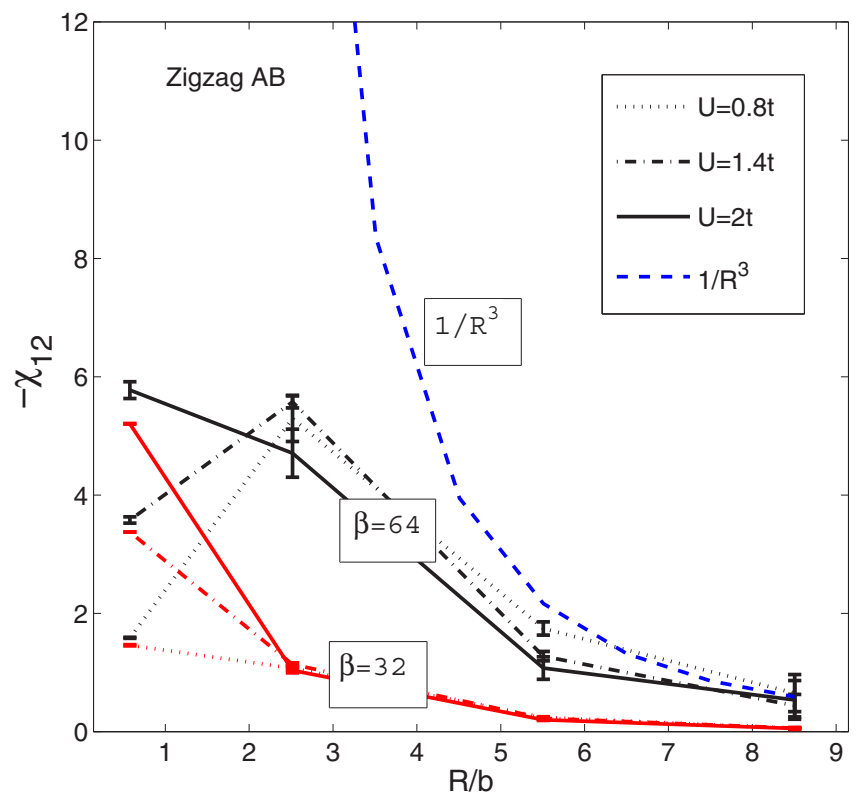

FIG. 2. (Color online) The static magnetic susceptibility between two magnetic adatom impurities along the zigzag direction as a function of distance for the $A B$ configuration obtained using the QMC method, at different inverse temperatures $\beta$ and different $U$. The dashed lines show the $R^{-3}$ decay predicted by the RKKY model. Even at the highest $U$ value, the QMC calculations yield a much longer-ranged effective magnetic coupling between the adatoms.
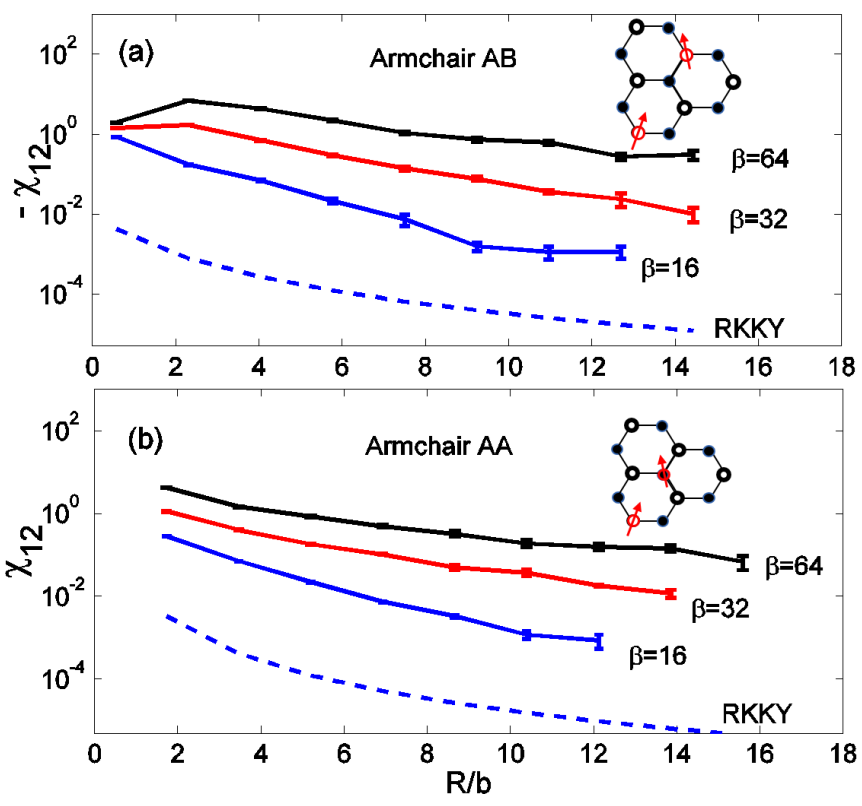

FIG. 3. (Color online) The static magnetic susceptibility between two magnetic adatom impurities along the armchair direction as a function of distance for (a) the $A B$ configuration (shown in the inset) and (b) the $A A$ configuration (shown in the inset), obtained by QMC calculations at different inverse temperatures $\beta$. The dashed lines are RKKY results from Ref. [19]. The magnetic coupling from QMC shows the same ferromagnetic and antiferromagnetic behavior. At low temperatures, the effective magnetic coupling becomes much stronger and the QMC results diverge from the RKKY's $R^{-3}$ decay.
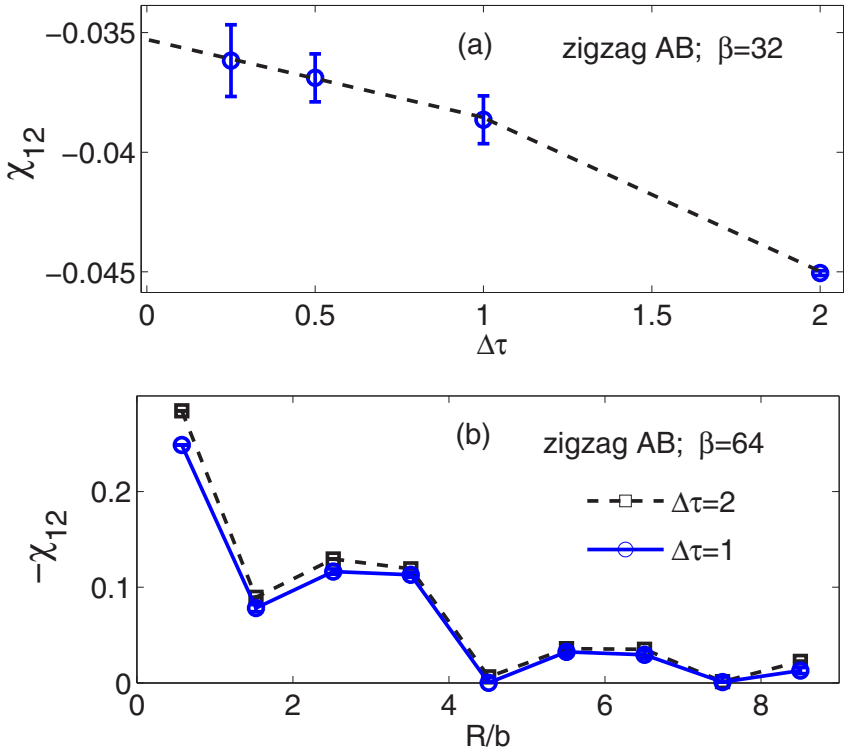

FIG. 4. (Color online) The static magnetic susceptibility between two magnetic adatom impurities along the zigzag for the $A B$ configuration obtained using the QMC method, at a different time step $\Delta \tau$, (a) for $\beta=32 t^{-1}$ and $R / b \sim 2.5$ (b) as a function of $R$ for $\beta=64 t^{-1}$. These results show that the finite time-step error is under control.

of the order $R / b \sim 10$, significantly deviating from the $R^{-3}$ behavior.

We note that the long-range behavior of the impurityimpurity correlations observed in our numerical results for the Anderson model is consistent with the predictions of Lieb's theorem for the Hubbard model in bipartite systems. According to Lieb's theorem [23], if there is an imbalance between the number of $A$ and $B$ sublattice atoms, a finite magnetic moment $\left(N_{A}-N_{B}\right) / 2$ arises at zero temperature. In our case, each impurity breaks the symmetry between the two sublattices locally. Thus, if the impurities are far from each other, locally a finite magnetic moment must appear at each impurity location. If the two impurities are on the same sublattices, the magnetic moments must add, or otherwise cancel each other out, giving rise to a strong ferromagnetic or antiferromagnetic interimpurity correlation.

We now discuss the finite time-step error involved in numerical calculations. In the QMC method, the partition function is discretized using $Z=\operatorname{Tr} \prod^{L} \exp (-\Delta \tau H)$, where $\Delta \tau$ is the size of the time step, $L$ is the number of Monte Carlo time slices, and $\beta=L \Delta \tau . Z$ defined above approaches the exact partition function of the system in the limit of infinite $L$, i.e., small $\Delta \tau$. In order to check the effect of using a finite time step, Fig. 4(a) shows $\chi_{i j}(R)$ for the third nearest $A B$ neighbors along the zigzag direction calculated for $\beta=64 t^{-1}$ using $\Delta \tau=2,1,0.5$, and 0.25 in units of $t^{-1}$. Actual calculations were done for $\Delta \tau=1$ in previous figures. The estimated time-step error is within a few error bars. We also plotted in Fig. 1(b) the results obtained for $\beta=64 t^{-1}$ using $\Delta \tau=2$ and 1 , showing the finite time-step error is under control in our calculations 


\section{CONCLUSION}

In conclusion, we studied the interaction between two magnetic adatom impurities in graphene within the Anderson model by using the quantum Monte Carlo technique. Our results yield to the same magnetic phases predicted by RKKY: ferromagnetic for the $A A$ (same sublattice) configuration and antiferromagnetic for the $A B$ (opposite sublattice) configuration. Moreover, $2 k_{F}$ oscillations similar to those of RKKY exist. However, the magnetic coupling between the impurities becomes more than two orders of magnitude stronger than what is predicted by the RKKY model, especially at lower temperatures. In addition, the results significantly diverge from the $R^{-3}$ decay predicted by RKKY and the effective interaction between the impurities become long ranged.

\section{ACKNOWLEDGMENTS}

This research was supported by a BIDEP grant from Türkiye Bilimsel ve Teknolojik Arastirma Kurumu (TÜBITAK), and by a BAGEP grant from Bilim Akademisi - The Science Academy, Turkey. The numerical calculations reported in this paper were partially performed at TÜBITAK ULAKBIM, High Performance and Grid Computing Center (TRUBA resources).
[1] P. R. Wallace, Phys. Rev. 71, 622 (1947).

[2] A. H. C. Neto, F. Guinea, N. M. R. Peres, K. S. Novoselov, and A. K. Geim, Rev. Mod. Phys. 81, 109 (2009).

[3] K. S. Novoselov, A. K. Geim, S. V. Morozov, D. Jiang, Y. Zhang, S. V. Dubonos, I. V. Grigorieva, and A. A. Firsov, Science 306, 666 (2004).

[4] Y. B. Zhang, Y. W. Tan, H. L. Stormer, and P. Kim, Nature (London) 438, 201 (2005).

[5] M. Wimmer, I. Adagideli, S. Berber, D. Tomanek, and K. Richter, Phys. Rev. Lett. 100, 177207 (2008).

[6] O. V. Yazyev and M. I. Katsnelson, Phys. Rev. Lett. 100, 047209 (2008).

[7] L. Yang, M. L. Cohen, and S. G. Louie, Phys. Rev. Lett. 101, 186401 (2008).

[8] J. Jung and A. H. MacDonald, Phys. Rev. B 79, 235433 (2009).

[9] F. Munoz-Rojas, J. Fernandez-Rossier, and J. J. Palacios, Phys. Rev. Lett. 102, 136810 (2009).

[10] B. Wunsch, T. Stauber, F. Sols, and F. Guinea, Phys. Rev. Lett. 101, 036803 (2008).

[11] S. Dutta, S. Lakshmi, and S. K. Pati, Phys. Rev. B 77, 073412 (2008).

[12] A. D. Güçlü, P. Potasz, O. Voznyy, M. Korkusinski, and P. Hawrylak, Phys. Rev. Lett. 103, 246805 (2009).

[13] P. Potasz, A. D. Güçlü, A. Wojs, and P. Hawrylak, Phys. Rev. B 85, 075431 (2012).

[14] A. D. Güçlü, M. Grabowski, and P. Hawrylak, Phys. Rev. B 87, 035435 (2013).

[15] S. R. Power and M. S. Ferreira, Crystals 3, 49 (2013).
[16] L. Brey, H. A. Fertig, and S. Das Sarma, Phys. Rev. Lett. 99, 116802 (2007).

[17] S. Saremi, Phys. Rev. B 76, 184430 (2007).

[18] A. M. Black-Schaffer, Phys. Rev. B 81, 205416 (2010).

[19] M. Sherafati and S. Satpathy, Phys. Rev. B 83, 165425 (2011).

[20] E. Kogan, Phys. Rev. B 84, 115119 (2011).

[21] B. Wunsch, T. Stauber, F. Sols, and F. Guinea, New J. Phys. 8, 318 (2006).

[22] B. Fischer and M. W. Klein, Phys. Rev. B 11, 2025 (1975).

[23] E. H. Lieb, Phys. Rev. Lett. 62, 1201 (1989).

[24] H. Sevincli, M. Topsakal, E. Durgun, and S. Ciraci, Phys. Rev. B 77, 195434 (2008).

[25] Y. G. Zhou, X. T. Zu, F. Gao, H. F. Lv, and H. Y. Xiao, Appl. Phys. Lett. 95, 123119 (2009).

[26] R. Xiao, D. Fritsch, M. D. Kuzmin, K. Koepernik, H. Eschrig, M. Richter, K. Vietze, and G. Seifert, Phys. Rev. Lett. 103, 187201 (2009).

[27] D. Jacob and G. Kotliar, Phys. Rev. B 82, 085423 (2010).

[28] T. O. Wehling, A. V. Balatsky, M. I. Katsnelson, A. I. Lichtenstein, and A. Rosch, Phys. Rev. B 81, 115427 (2010).

[29] K. T. Chan, H. Lee, and M. L. Cohen, Phys. Rev. B 83, 035405 (2011).

[30] A. N. Rudenko, F. J. Keil, M. I. Katsnelson, and A. I. Lichtenstein, Phys. Rev. B 86, 075422 (2012).

[31] A. Saffarzadeh and G. Kirczenow, Phys. Rev. B 85, 245429 (2012).

[32] J. E. Hirsch and R. M. Fye, Phys. Rev. Lett. 56, 2521 (1986).

[33] F. M. Hu, T. Ma, H.-Q. Lin, and J. E. Gubernatis, Phys. Rev. B 84, 075414 (2011). 